\title{
Eine Diskussion zur Datenschutz-Grundverordnung
}

\author{
Peter Mertens ${ }^{1}$ \\ (c) Springer-Verlag GmbH Deutschland, ein Teil von Springer Nature 2020
}

Der Datenschutz steht schon lange im Zielkonflikt mit anderen gesellschaftlichen und wirtschaftlichen Anliegen.

Nach Verabschiedung des Bundesdatenschutzgesetzes 1977 sahen viele eine zu starke Rücksichtnahme auf Verbrecherinnen und Verbrecher (,Datenschutz oder Tatenschutz?"). Zwischen 1990 und 2012 folgten öffentliche Diskussionen zu Datenschutz und Informationsfreiheit im Zusammenhang mit der Geheimhaltung der persönlichen Daten von begnadigten RAF-Mitgliedern, zur spektakulären Darstellung der Verhaftung eines später verurteilten prominenten Steuer-Straftäters in einem öffentlich-rechtlichen Sender und zum Ankauf von gestohlenen „SteuerCDs".

Aktuell liegt der Zielkonflikt zwischen Datenschutz und Gesundheitsschutz offen.

In der gegenwärtigen politischen Diskussion befinden sich Erwägungen, z. B. des deutschen Bundeswirtschaftsministers, besondere Maßnahmen zu ergreifen, um den Unternehmen „nach Corona“ die Rückkehr zur Normalität zu erleichtern und dazu auch die Verwaltungsgemeinkosten zu senken sowie Führungskräfte zu entlasten. In einem zweijährigen Moratorium sollen demnach die deutschen Gesetzgeber und die EU-Kommission keine neuen mit beträchtlichem Arbeitsaufwand verbundenen Vorschriften erlassen und bereits vorhandene entschärfen (,Belastungsmoratorium“). Das würde voraussichtlich auch den Datenschutz betreffen, zumal nicht nur Privatpersonen und Unternehmen, sondern auch Datenschutz-Behörden außerordentlich belastet sind.

Mit dem vorliegenden Heft will das Informatik Spektrum die Diskussion um die DSGVO aus verschiedenen Blickwinkeln bereichern. Wir freuen uns, dass auch der Bundesdatenschutzbeauftragte (er ist Diplominformatiker und Honorarprofessor an einem Hochschulzentrum für Ethik und Verantwortung) trotz seiner extremen Arbeitslast teilnimmt,

\footnotetext{
Peter Mertens

peter.mertens@fau.de

1 Universität Erlangen-Nürnberg, Erlangen, Deutschland
}

dazu ein Rechtswissenschaftler, der sich dem Datenschutz schon sehr früh zugewandt hatte, ein kaufmännischer Leiter eines mittelständischen Unternehmens und ein Vertreter des relativ neuen Berufsstands der externen Datenschutzbeauftragten. Dazu komme ich als Wirtschaftsinformatiker.

Alle Autoren sind prinzipiell Befürworter des Datenschutzes, wenn auch mit unterschiedlichen Positionen zu Einzelheiten der Datenschutz-Grundverordnung.

\section{Anhang}

\section{Zum Titelbild}

Zur Erkennung manipulierter Bilder und „Deep Fake“Videos müssen Detektoren authentisches Material von synthetischem unterscheiden. Diese Synopse stellt ein

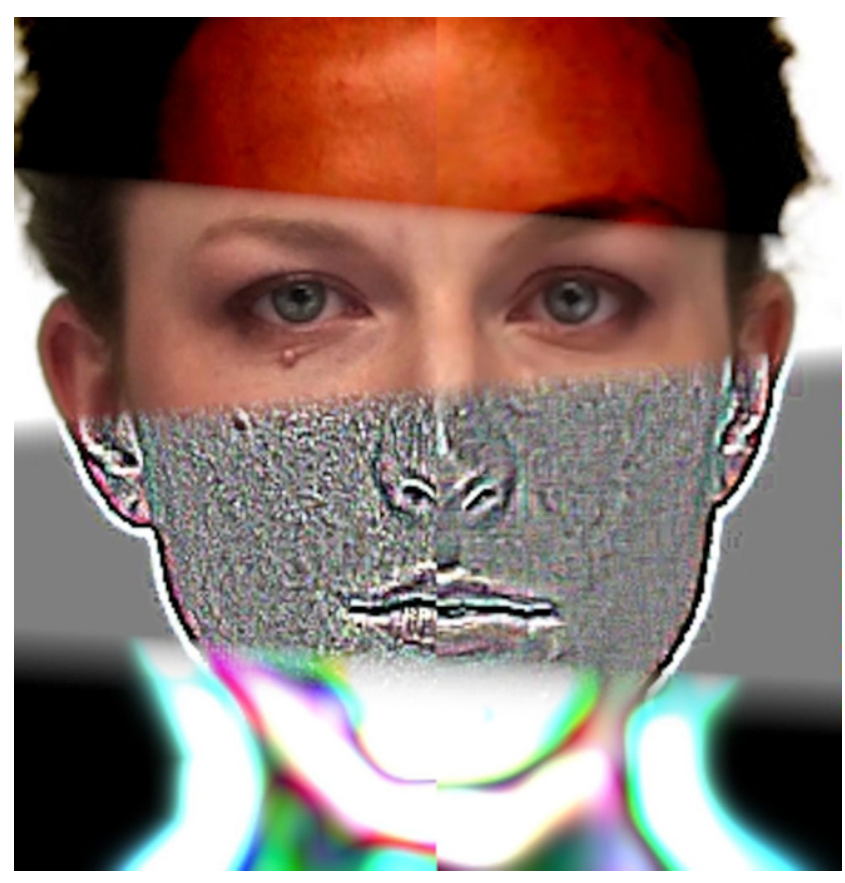

Abb. 1 Automatic forgery detection 
authentisches Bild (linke Hälfte) einer synthetischen Rekonstruktion (rechte Hälfte) gegenüber, jeweils nach Anwendung verschiedener Filter: Die Farbverteilung über der Stirn wurde normalisiert und beschnitten. Der Bereich vom Kinn abwärts wurde einem temporalen Filter unterzogen. Die Augenpartie wurde nicht verändert. Obwohl die Rekonstruktion (rechts) fast makellos scheint, fördert der räumliche High-Pass-Filter für die Nase und Wangen einen klaren Unterschied zum authentischen Bild zutage. Zukünftige Synthese-Algorithmen werden solche Imperfektionen vermeiden, was Detektoren vor neue Probleme stellt. Das authentische Rohbild stammt aus der Ryerson Audio-Visual Database of Emotional Speech and Song (RAVDESS). Die Filter basieren auf VideoForensicsHQ (Fig. 1).

Gereon Fox, B.Sc.

Doctoral Researcher

Max Planck Institute for Informatics

Saarland Informatics Campus

Campus E1 4

66123 Saarbrücken

http://people.mpi-inf.mpg.de/ gfox/

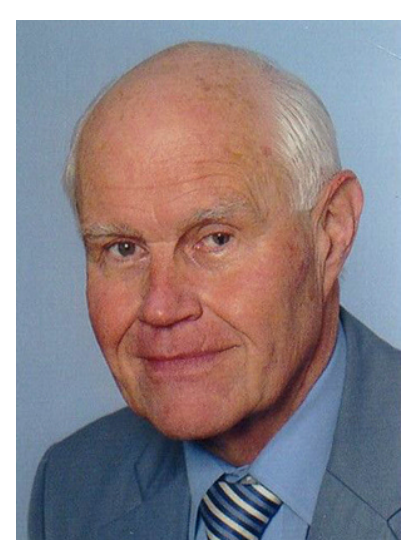

Peter Mertens arbeitet als emeritierter Professor am Lehrstuhl Wirtschaftsinformatik 1 der Universität Erlangen-Nürnberg. 